\title{
Merging of Flight Test Data within the UMAT TDS
}

\author{
Tjorven Gerhard ${ }^{1}$, Tobias Paul ${ }^{1}$ \\ ${ }^{1}$ ESG Elektroniksystem- und Logistik GmbH, Fürstenfeldbruck, Germany \\ tobias.paul@esg.de
}

\begin{abstract}
:
In close cooperation with Bundeswehr, ESG has modified an UMS Skeldar R-350 UAS to offer a testbed for in flight evaluation of payload and avionics. This testbed is called Unmanned Mission Avionics Test Helicopter (UMAT). In order to provide a complete test environment, a truck based laboratory enhances monitoring, control, and recording capabilities. It is called Flexible Mobile Ground Control Station (FlexMobGCS). In this paper we present the UMAT Test Data System (TDS) which is a modular, digital and powerful system for capturing, recording, transmitting, analysing and archiving of test data during flight test operations with the UMAT. The TDS is used to evaluate experimental equipment during flight tests like avionics or mission management functions as well as human machine interactions. The TDS consists of an air segment within the UMAT, a data link and the ground segment within the FlexMobGCS. In this paper the focus lies on the challenges of the TDS ground segment and its integration into the TDS air segment.
\end{abstract}

Key words: testbed, unmanned aircraft, avionics, recording

\section{Introduction}

The UMAT [1], [2] is based on the R-350 VTOL Remotely Piloted Air System (RPAS) of UMS Skeldar, see Fig. 1. The R-350 has a rotor diameter of $3.5 \mathrm{~m}$ and a maximum take-off weight of $145 \mathrm{~kg}$ including a payload weight of $38 \mathrm{~kg}$. The maximum flight duration is estimated with $3.5+\mathrm{h}$ at a maximum speed of $120 \mathrm{~km} / \mathrm{h}$. Due to the fact that the base systems is part of a continuous development, the flight envelope might vary in future.

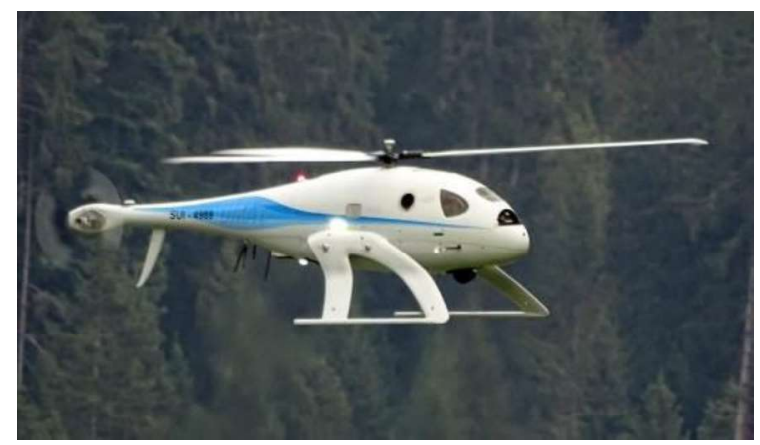

Fig. 1. UMAT

To provide a test helicopter, modifications to the purchased R-350 have been made. With these modifications, experimental equipment can be tested either mounted on a nose payload bay carrier or within a cassette which is mounted in the R-350s main payload bay. Both, nose payload bay carrier and the cassette are mechanically and electrically decoupled from the $\mathrm{R}-350$. In addition, the $\mathrm{R}-350$ is protected by a firewall like equipment toward erroneous signals submitted by the experimental equipment. Those extensions allow the flight testing of not certified / qualified experimental equipment.

The capabilities of the UMAT focus on four areas:

1. Evaluation of performance capabilities of alternative COTS (commercial of the shelf) mission avionics

2. Risk reduction during development, e.g. by bringing rapid prototypes into flight test

3. Examination of new mission concepts like MUM-T [3] (Manned-Unmanned Teaming) or SAAFu [4] (Sense \& Avoid Assistance Function for the UAS Operator)

4. Examination of certification topics and operating procedures

Within those areas ESG performs the integration, performs or supports the steps: flight test planning, execution of the flight tests, and data analysis.

The available state-of-the-art experimental Mission Management Computer - based on an open Integrated Modular Avionics (IMA) [5], [6] architecture - supports the development and analysis of mission management functions, that will boost the autonomy of future UAS. As a 
tactical-UAS-sized system the UMAT is ideally suited to support the demonstration of operational concepts for military and civil VTOL-UAS applications.

The Flexible Mobile Ground Control Station (FlexMobGCS, see Fig. 2) consists of the workstations for the pilot in command (PIC) and the Payload Operator (PO) in the front, including redundant mission computers and flight safety management computers. Additionally the FlexMobGCS includes two flight test engineer workstations in the forward section. The rear section holds the flight test observation area where the flight test can be monitored by customers, see Fig. 3. This rear section can also be converted to a maintenance and assembling area for the UMAT. In addition this rear part is used to transport the UMAT to the flight test area.

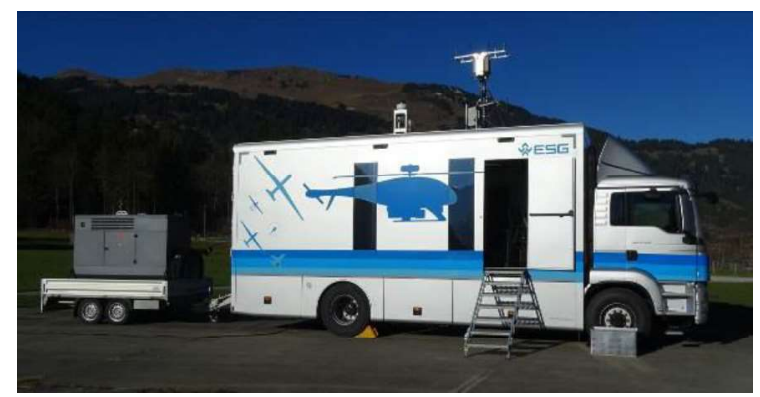

Fig. 2. FlexMobGCS with generator

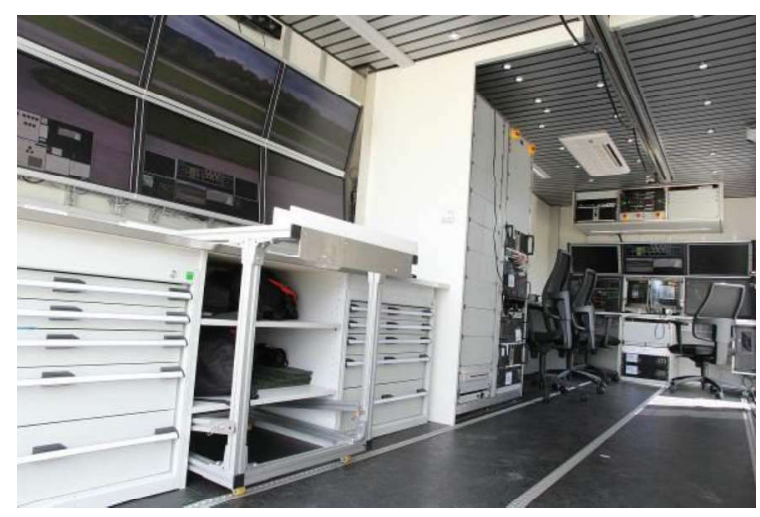

Fig. 3. FlexMobGCS interior

\section{The Test Data System}

The UMAT Test Data System (TDS) is a modular system which enables the flight test engineer to perform a live analysis during the flight test as well as after the flight. With the TDS, the system state and flight state data of the UAS are captured as well as data of the permanent test equipment including HumanMachine-Interactions (HMI) and the variable flight test equipment that may differ for every flight test. To be able to address all these areas, a digital and powerful system for capturing, recording, transmitting, analysing, and archiving of test data during flight test operations with the UMAT is needed, see Fig. 4. Some test data is captured and recorded on board the UMAT or sent down to the ground control station via the TDS data link whereas other test data is captured in the ground control station. Each relevant data source in the UMAT and the FlexMobGCS is captured. The captured data is then transmitted to recording devices and to analysis software. After the flight test, they can be transmitted to a data server for further processing or archiving.

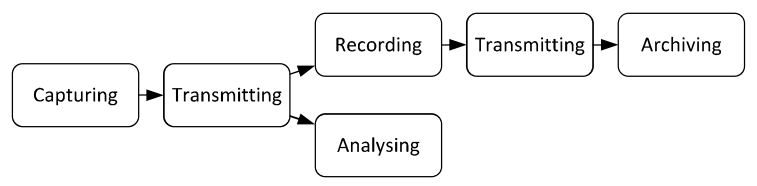

Fig. 4. TDS functions

The test data system consists of three segments:

- the air segment which includes the capturing and recording of data in the UMAT,

- the data link which sends the test data from the UMAT live to the FlexMobGCS, and

- the ground segment which includes all data sources in the FlexMobGCS as well as the analysis software and the archiving capabilities.

For the air segment of the test data system, several commercial solutions were available using manned test flight equipment. In general, a test data system for manned aircrafts can also be used for the UMAT if size and weight are within the payload capabilities. Using a test data system for a manned aircraft has the advantage of choosing complete and mature solutions. The requirements for the TDS of the air segment include: reliability, rugged design, and weather resistant. Some of the available solutions were: PowerDNA from United Electronics Industries, HiDANplus from RTD Embedded Technologies, KAM-500 from ACRA CONTROL, and MiniR 700 from AMPEX. For the UMAT we chose the KAM-500 from ACRA Control as the TDS air segment because it is reliable, offers many different sensors for the air segment and analysis software is available.

Regarding the required high capacity test data link, commercial state of the art solutions were available also. These solutions include but are not limited to: Condor UAV RF Tranceiver from Sabtech, Airborne Transmitter and Receiver from Emhiser, X-5000-REC from Smartronix, and Digital Data Link from NSM Surveillance. We chose the NSM Surveillance data link. 
The ground segment of the UMATs test data system had to be integrated into the analysis software of the air segment, so that data from the ground segment can be analysed together with data from the air segment. The data sources in the FlexMobGCS are mostly COTS components and require modification to be compatible with the test data system of the air segment. As a result there are no state of the art solutions for the ground segment available. Instead customized solutions had to be found. The following data sources and sensors in the FlexMobGCS have to be addressed by the ground segment of the test data system:

- Weather station: A weather station is installed outside the GCS to capture among other things temperature, air pressure, wind speed and direction.

- GCS internal temperatures: Additionally the temperature inside the GCS is captured.

- Cameras: Several cameras are mounted in and outside the FlexMobGCS to monitor the interior of the GCS, the landing site and to track the UMAT during flight.

- Data links: Furthermore the network communication of the data links is monitored. There are three different data links in the FlexMobGCS:

○ the command and control (C2) data link,

- the video data link for the camera mounted on the UMAT and

○ the TDS data link.

- Network communication: Additionally the network communication of the PIC and PO workstations including the mission computers, the flight safety management computers and the ground data terminals is captured.

- HMI: For the analysis of human machine interactions and human factors the input of the PIC and Po via keyboard, mouse or rotary switches into the mission and flight safety management computers has to be captured as well as the monitor screens of these computers. Head movement and movement of PIC and PO are also information that are captured for analysing human machine interaction and human factors.

- Intercom system: Another data source that has to be addressed is the intercom system in GCS.
As a result many different signals have to be captured including USB, serial, Ethernet, DVI and analogue signals.

\section{Faced Challenges}

In the following section, the challenges und solutions of the TDS for the ground segment as well as the challenges resulting from the integration into the analysis software of the air segment are presented. The TDS for the ground segment has to handle challenges like:

1. Time synchronization of the air and ground segment

2. Integration of the ground data sources into the analysis software of the air segment

3. Expandability of the ground system for future experimental equipment which is unknown today

4. Different data types have to be captured and processed ranging from huge video data or audio data to high frequency numerical data.

5. Controlling a distributed system.

Both the systems in the FlexMobGCS and the UMAT are time synchronized with GPS so that data is perfectly synchronized when it is captured. The analysis software runs in the GCS so that the data from the GCS is nearly instantly in the analysis software. The data from the UMAT has to be send down to the GCS via the TDS data link and thereby arriving at the analysis software delayed. As a result, the data of the ground and the air segment is no longer synchronized which poses a problem for the analysis software because it can only cope with time synchronized data where $t_{2}=t_{3}$ is satisfied in Fig. 5. The only solution is to resynchronize the data by either artificially delaying the data from the ground segment or by overriding the timestamp of the air data in the ground segment and sending the air data as well as the value of the time delay to the analysis software.

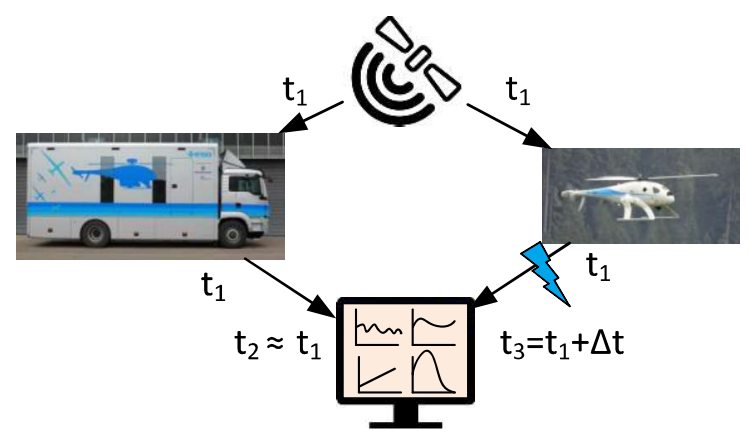

Fig. 5. Time synchronization of the ground and air segment 
To integrate the ground segment into the air segment the data of the ground segment has to be analysed with the analysis software of the air segment. The analysis software provides an interface for numerical data. This Ethernet interface accepts numerical data in the proprietary IENA format. The data sources in the ground control station that generate numerical data provide different interfaces ranging from proprietary Ethernet over serial to USB interfaces. In order to convert the data into the IENA format for each data source, a customized software module is implemented to convert the data. If necessary, a hardware capturing device is connected to the data source so that the customized software can access the data. The data is then forwarded to the analysis software as well as a recording software module as shown in Fig. 6 .

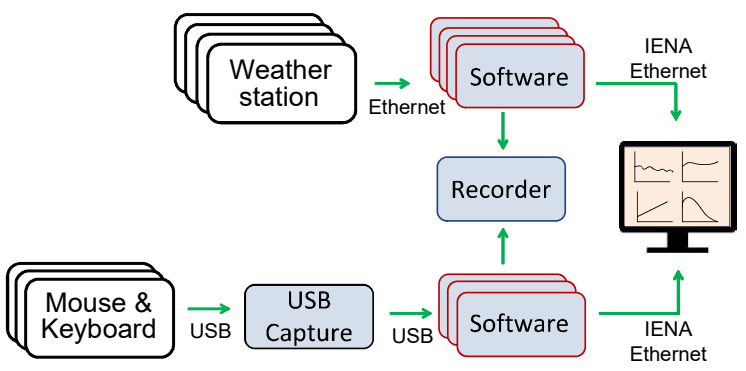

Fig. 6. Integration of numerical data

For the multimedia data sources in the ground segment like the intercom, the computer screens, and the cameras, a different solution is needed. Due to the huge data sizes of the multimedia data, the analysis software of the air segment is not suited for the analysis of this data. As a result, a second analysis software optimized for multimedia data is implemented. Both analysis software have to be synchronized to enable the flight test engineer to analyse numerical and multimedia data together. This means that an interface between the air segment analysis software and the multimedia analysis software has to be implemented so that the multimedia analysis software can be controlled by the analysis software of the air segment.

The test data system has a modular design to prepare the system for future expansion due to new experimental equipment in the air segment or the ground segment. New experimental equipment in the air segment can either be integrated for on-board capturing and recording into the sensor suite of the test data system of the air segment (KAM-500) or the data of the new experimental equipment can be sent to the ground segment for capturing and recording in the ground segment. For capturing and recording of new experimental equipment in the ground segment, where the data source itself can either be in the air segment or in the ground segment, the modular TDS can be expanded by writing a new customized software module for the new data source and adding if necessary a capturing device as depictured in Fig. 6.

With the architecture in Fig. 6, sources with different data types can be realized in the Test Data System. If huge data sizes occur, which are the cases when capturing high resolution video or DVI data, the capturing device has to be able to handle them by reducing the frequency and thus not capturing every frame and by compressing the video using standard algorithms like the H.264.

The ground segment of the test data system is a highly distributed system where hardware capturing modules are distributed throughout the FlexMobGCS and where the customized software modules are running on different computers and servers, see Fig. 7 . The flight test engineer has a dedicated workstation from which he can control all hardware and software components of the test data system. We have implemented a control software for the TDS which provides the flight test engineer with a graphical user interface to control the TDS ground segment. Using this software, the flight test engineer can

1. Configure all hardware and software components

2. Start and stop all TDS functions on the hardware components

3. Transfer all recorded data from every hardware to a data server

4. Shutdown all hardware components

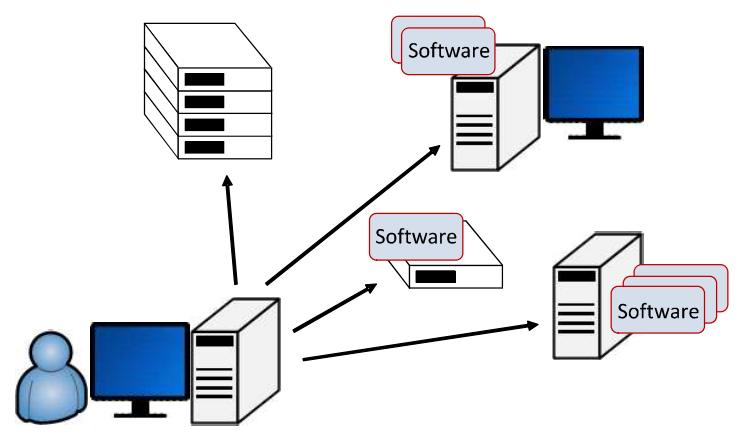

Fig. 7. The distributed system

\section{Conclusion}

A test data system for an unmanned aircraft system consists of three segments:

- the air segment,

- the data link, and

- the ground segment. 
All three segments are integrated in the test data system and function as one system. The test data system is used to analyse the flight test. Therefore it has to capture, record, transmit, analyse, and archive the test data. For the air segment solutions from the manned test aviation can be integrated in the unmanned aircraft and for the data link segment available digital data links can be used.

There is no commercial of the shelf solution available regarding the ground segment because the COTS components of the ground control station cannot easily be integrated with the aircraft components of the air segment. For this integration to work customized software for each data source in the ground segment has to be implemented to convert the data. The following challenges have to be addressed when integration ground and air segment: the time synchronization of air and ground segment, the integration of test data from ground and air segment into one analysis software, the expandability of the system for future experimental equipment that is unknown today, the management of different data sources and different data formats and the control of the distributed system by the flight test engineer. In this paper solutions for these challenges are presented.

\section{References}

[1] H.-W. Schulz, Certification Process for a VTOLUAS Experimental System, 26th International UAV Systems Conference, Bristol / UK, 2011.

[2] H.-W. Schulz, The Unmanned Mission Avionics Test Helicopter - A flexible and versatile VTOLUAS Experimental System, International Archives of the Photogrammetry, Remote Sensing and Spatial Information Science, Zürich / CH, 2011.

[3] T. Paul und E. Brämer, Operational Considerations for Teaming Manned and Unmanned Helicopter, 2012 International Conference on Unmanned Aircraft Systems, Philadelphia / USA, 2012.

[4] O. Horz, Integration of Military Unmanned Aircraft Systems Into German Airspace \& Sense and Avoid Assistant, DGLR Symposium 2013: Workshop "UAV Autonomie" - Automatisierung unbemannter Lufffahrzeuge, München, 2013.

[5] E. Brämer, IMA - Integrierte Modulare Avionik, Spektrum - Magazin der ESG Elektroniksystemund Logistik-GmbH, pp. 6-7, Oktober 2010.

[6] M. Gangkofer, IMA ${ }^{3}$, Spektrum - Magazin der ESG Elektroniksystem- und Logistik-GmbH, pp. 5-6, Juli 2012. 